\title{
Application of the Difference Threshold Test on Asynchronous BFSK Frequency-Hopped Multiple Access Systems
}

\author{
Ing-Jiunn $\mathrm{Su}$ and Jingshown $\mathrm{Wu}$ \\ Room 519 \\ Department of Electrical Engineering \\ National Taiwan University \\ Taipei, Taiwan 10617, China \\ TEL: 886-2-23635251-519 \\ FAX: 886-2-23638247 \\ E-mail: ijsu@r528d.ee.ntu.edu.tw
}

\begin{abstract}
The new side information generating mechanism, difference threshold test (DTT), is proposed to improve the capacity of asynchronous frequency-hopped multiple ac(cess system with binary frequency shift keying (BFSK) signaling over Rayleigh fading channels. By adjusting the threshold in the DTT decision logic, the optimum capacity ran be obtained and proved advantageous over that with conventional hard decision. For the systems with signal-to-noise ratio ranging from 10 to $13 \mathrm{~dB}$, this improvement (an be over $12 \%$.
\end{abstract}

\section{Introduction}

Frequency-hopped multiple access (FHMA) techniques have attracted increasing interests in the application of wireless communications. However the simultaneously transmitted signals may collide in the same frequency band, called as hit events. The hit symbols would be liable to make wrong decision for the hard decision scheme at the receiver. If a side information generating mechanism can indicate this event and inform the following decoder to erase these hit symbols, the performance would be improved. In the literature [1]. some schemes are proposed to provide this side information on the FHMA systems. In this paper we propose a new scheme, called as difference threshold test (DTT), to generate this side information. DTT scheme just performs comparison operations in decision logic: which is simpler than that in the conventional side information generating mechanisms, and would simplify the receiver circuit design. This paper will investigate its effectiveness in the asynchronous binary frequency shift keying (BFSK) FHMA system over Rayleigh channels. Concoherent detection scheme is assumed in this paper.

\section{System description and analysis}

We consider that $K$ users are active in the FHMA system allocated with $q$ available frequency slots. Each transmitter modulates information bit with BFSK signaling scheme. The 
carrier frequency of each modulation symbol is changed with respective random sequence from available frequency slots. With no coordination in user hopping sequences, the transmitted symbols may be hit by other simultaneously transmitted signals. The probability of symbol being hit by a specific user signal in asynchronous hopping system can be represented by:

$$
p_{h} \simeq \frac{2}{q}
$$

The receiver first wipes the frequency hopping effect from the received signal according to the intended hopping pattern and direct it to envelope detectors. For BFSK signaling, two envelope outputs are generated for decision and represented by [2]:

$$
r_{i}=\left|Z_{i}+\delta_{i 0} \alpha_{0} e^{j \theta_{0}}+\sum_{m=1}^{K-1} C_{i m} \alpha_{m} x_{m} e^{j \theta_{m}}\right|, \quad i=0,1
$$

where $\delta_{i 0}$ is Kronecker delta, $Z_{i}$ is the complex Gaussian noise with zero mean and $E\left\{Z_{i} Z_{i}^{*}\right\}=$ $N_{0} / 2, N_{0}$ is one-side power spectral density of noise, $C_{i m}$ is a binary-value random variable to represent whether the $m$-th user presents signal in the $i$-th envelope output, $\alpha_{m}$ and $\theta_{m}$ are the amplitude and phase of the $m$-th user signal, and $x_{m}$ is a random variable uniformly distributed over $[0,1)$ to reflect partial hit nature in asynchronous hopping systems. Here we assumed that the 0 -th user signal is intended to receive and the envelope output $r_{0}$ contains the signal of the 0 -th user. For Rayleigh channels, $\theta_{m}$ is uniformly distributed over $[0,2 \pi)$ and $\left\{\alpha_{m}\right\}$ are modeled as independent and identically distributed (i.i.d.) Rayleigh random variables with variance $2 \sigma_{f}^{2}$ by assuming same average power for all user signals. The probability function of $C_{i m}$ can be represented by:

$$
\operatorname{Pr}\left(C_{i m}\right)= \begin{cases}\frac{p_{h}}{2}, & C_{i m}=1 \\ 1-\frac{p_{h}}{2}, & C_{i m}=0\end{cases}
$$

The corresponding characteristic functions of two envelope outputs can be represented as [3],[4]:

$$
\phi_{i}(\rho)=e^{-\frac{\left(\sigma^{2}+\delta_{i 0} \sigma_{f}^{2}\right) \rho^{2}}{2}} \prod_{m=1}^{K-1} I\left(C_{i m} \rho\right),
$$

where

$$
I(\rho)=\int_{0}^{1} e^{-\frac{x^{2} \sigma_{f}^{2} \rho^{2}}{2}} d x
$$

is the average characteristic function of interference signal component. If $K$ goes infinity with $\lambda=K / q$ held constant, Poisson approximation can be applied to $C_{i m}$ and the average characteristic functions can be found [5]:

$$
\hat{\phi}_{i}(\rho)=e^{-\frac{\left(\sigma^{2}+\delta_{i 0} \sigma_{f}^{2}\right) \rho^{2}}{2}} e^{\lambda[I(\rho)-1]}
$$

By inverse transformation, the probability density functions, $f_{i}(r)$, and cumulative distribution functions, $F_{i}(r)$, can be found to evaluate the performance.

For the DTT decision scheme, a threshold $d$ is preset to estimate the reliability of received symbol. If the difference between two envelope outputs is smaller than this threshold, a 
crasure side information is issued to indicate unreliable receiving. Otherwise the decision logic chooses the largest output as the estimated symbol. More erasure side information would be generated for larger threshold. If the threshold is set to zero, no side information ran be generated and the decision scheme is degenerated as hard decision. According to this decision rule, the correction decision occurs when non-desired envelope output $r_{1}$ is less than $r_{0}-d$ and its probability is determined by [4]:

$$
P_{c}=\int_{0}^{\infty} F_{1}(r-d) f_{0}(r) d r
$$

Similarly the error probability can expressed as:

$$
P_{e}=\int_{0}^{\infty} F_{0}(r-d) f_{1}(r) d r
$$

For the symmetrical binary erasure and error channel model, the total channel capacity, in terms of bits per channel use. can be derived as:

$$
C=K\left\{\dot{P}_{c} \log _{2} \frac{2 P_{c}}{1-P_{x}}+P_{e} \log _{2} \frac{2 P_{e}}{1-P_{x}}\right\}
$$

where $P_{t}=1-P_{c}-P_{e}$ is the erasure probability.

\section{Numerical results}

Since the threshold affects the generation of side information, the system performance would be varying with threshold setting. Although more erasure side information can be grnerated for larger $d$, the excess erasure would just waste useful information and reduce the system capacity: Therefore there exists an optimum threshold maximized the capacity for 'ach user density: With numerical search. we can find this optimum threshold for each user density. Fig. 1 presents the maximum capacity and optimum thresholds for $E_{b} / N_{0}=10$ $\mathrm{dB}$ and $E_{b} / \wedge_{0}=13 \mathrm{~dB}$. where $E_{b}=2 \sigma_{f}^{2}$ is the average bit energy. Here the threshold is nomalized to $\sqrt{E_{b}}$. As intuition. the threshold should be increased for larger user density due 10 more hit rrents. In this figure, the results for hard decision $(d=0)$ are also presented to illustrate the effectiveness of side information. In general, the system with large $E_{b} / N_{0}$ would have more capacity regardless of decision scheme. Moreover it is found that the capacity with optimum threshold in DTT decision can be significantly improved over that with hard derision. Over $12 \%$ improvement in maximum attainable capacity can be attainable.

\section{Conclusion}

A new DTT decision scheme is proposed for generating erasure side information in the asynchronous BFSK FHMA system over Rayleigh fading channels. This scheme simplifies the operation in decision logic and reduces the complexity of implementation. By optimizing the threshold of DTT decision logic, the generated side information can effectively improve sistem performance. Compared with hard decision systems, the maximum attainable caparity for the DTT decision system can be improved over $12 \%$ when the signal-to-noise ratio ranges from 10 to $13 \mathrm{~dB}$. It is also revealed that the optimum threshold should be increased for larger user density. 


\section{Réferencés}

[1] M. B. Pursley, "The derivation and use of side information in frequency-hop spread spectrum communications." IEICE Trans. Commun,, vol. E76-B, pp. 814-824, Aug. 1993.

[2] I.-J. Su and J. Wu, "Capacity analysis of the BFSK FHMA system with RTT side information generation in fading channels," submitted for publication in IEEE Comm. Lett.

[3] J. S. Bird, "Error performance of Binary NCFSK in the presence of multiple tone interference and system noise," IEEE Trans. Commun., vol. 33, pp. 203-209, Mar. 1985.

[4] J. S. Bird and D. A. George, "The use of the Fourier-Bessel series in calculating error probabilities for digital communication systems," IEEE Trans. Commun., vol. 29, pp. 1357-1365, Sep. 1981.

[5] I.-J. Su and J. Wu, "Performance of asynchronous slow-frequency-hopped multiple access systems with RTT techniques for side information," IEICE Trans. Fundamentals, vol. E81-A, No. 2, pp. 327-332, Feb. 1998

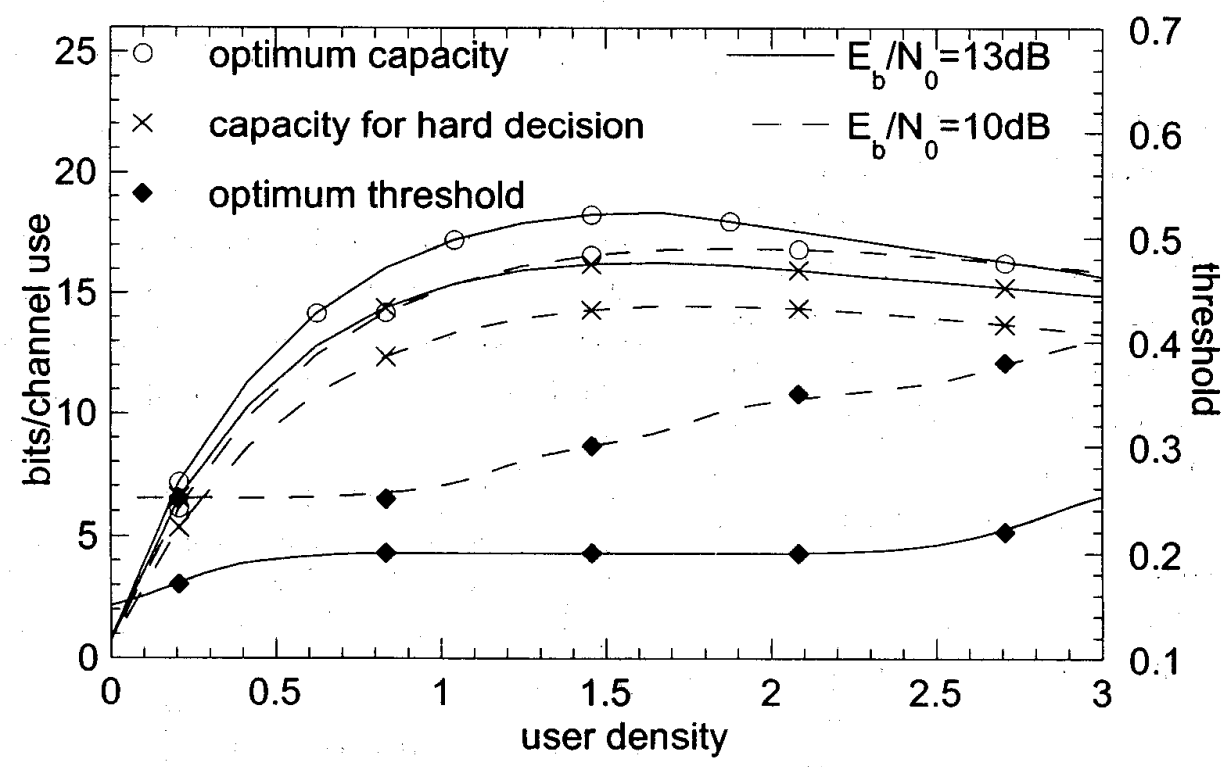

Fig. 1 Capacity and optimum threshold 